\title{
ANALYTIC EXTENSIONS AND SELECTIONS ${ }^{1}$ BY
}

\author{
J. GLOBEVNIK
}

\begin{abstract}
Let $G$ be a closed subset of the closed unit disc in $C$, let $F$ be a closed subset of the unit circle of measure 0 and let $\Phi$ map $G$ into the class of all open subsets of a complex Banach space $X$. Under suitable additional assumptions on $\Phi$ we prove that given any continuous function $f: F \rightarrow X$ satisfying $f(z) \in \operatorname{closure}(\Phi(z))(z \in F \cap G)$ there exists a continuous function $f$ from the closed unit disc into $X$, analytic in the open unit disc, which extends $f$ and satisfies $\tilde{f}(z) \in \Phi(z)(z \in G-F)$. This enables us to generalize and sharpen known dominated extension theorems for the disc algebra.
\end{abstract}

Let $p$ be a real valued positive continuous function on the unit circle $T$ in $C$ and let $F \subset T$ be a closed set of Lebesgue measure zero. Given any continuous function $f: F \rightarrow C$ satisfying $|f(s)|<p(s)(s \in F)$ there exists a function $\tilde{f}$ in the disc algebra which extends $f$ and satisfies $|\tilde{f}(t)|<p(t)$ $(t \in T)$. This simple dominated extension theorem is a special case of a more general theorem proved by E. Bishop [1]. See [1] [3], [6], [7], [10] for such theorems in general spaces of continuous functions and see [7] for the most general dominated extension theorem in the disc algebra.

Writing $\Phi(t)=\{z \in C:|z|<p(t)\}(t \in T)$ the above theorem becomes a selection theorem: Given any continuous function $f: F \rightarrow C$ satisfying $f(s) \in$ $\Phi(s)(s \in F)$ there exists a function $\tilde{f}$ in the disc algebra which extends $f$ and satisfies $\tilde{f}(t) \in \Phi(t)(t \in T)$.

In the present paper we use some ideas of [5] to prove a selection theorem for the disc algebra which generalizes and sharpens known results on dominated extensions.

Throughout, we denote by $\Delta, \bar{\Delta}$ and $\partial \Delta$ the open unit disc in $C$, its closure and its boundary, respectively. If $X$ is a complex Banach space and $r>0$ we write $B_{r}(X)=\{x \in X:\|x\|<r\}$. Let $x \in X$ and $S, T \subset X$. We write $x+S$ $=\{x+u: u \in S\}$ and $S+T=\{u+v: u \in S, v \in T\}$ and denote by $\bar{S}$ the closure of $S$. By $A(\Delta, X)$ we denote the Banach space of all continuous functions from $\bar{\Delta}$ to $X$ which are analytic on $\Delta$ and by $A$ we denote the disc algebra $A(\Delta, C)$. We write $I=\{t: 0 \leqslant t \leqslant 1\}$ and denote the set of all positive integers by $N$.

Received by the editors April 20, 1978 and, in revised form, September 20, 1978.

AMS (MOS) subject classifications (1970). Primary 30A80, 30A96, 46J15.

${ }^{1}$ This work was supported in part by the Boris Kidric Fund, Ljubljana, Yugoslavia. 
Suppose that $\left\{p_{\alpha} ; \alpha \in \mathbb{Q}\right\}$ is a family of nonempty open subsets of $X$. For each $\alpha \in \mathbb{Q}$ let $x_{\alpha} \in \bar{P}_{\alpha}$. We say that the sets $P_{\alpha}(\alpha \in \mathbb{Q})$ are equi-locally connected at the points $x_{\alpha}$ if given any $\varepsilon>0$ there is some $\delta>0$ such that for every $\alpha \in \mathbb{Q}$ the set $\left(x_{\alpha}+B_{\delta}(X)\right) \cap P_{\alpha}$ is contained in a connected component of $\left(x_{\alpha}+B_{\varepsilon}(X)\right) \cap P_{\alpha}[9]$. We call any such $\delta(\cdot)$ a modulus of equi-local connectedness of the sets $P_{\alpha}(\alpha \in \mathbb{Q})$ at the points $x_{\alpha}$.

Let $S \subset \bar{\Delta}$ be a closed set and let $X$ be a Banach space. We call the graph of a map $\Phi: S \rightarrow 2^{X}$ the set of all pairs $(z, x) \in S \times X$ such that $x \in \Phi(z)$. We say that $\Phi$ is open if its graph is open in $S \times X$; equivalently, $\Phi$ is open if given any $z \in S$ and any $x \in \Phi(z)$ there is some $\varepsilon>0$ such that $x+B_{\varepsilon}(X)$ $\subset \Phi(u)(u \in S:|u-z|<\varepsilon)$. In particular, if $\Phi$ is open then $\Phi(z)$ is open for every $z \in S$.

Our main result is the following:

THEOREM. Let $X$ be a complex Banach space and let $G \subset \bar{\Delta}$ be a closed set. Assume that $\Phi: G \rightarrow 2^{X}$ is an open map such that $g(z) \in \Phi(z)(z \in G)$ for some $g \in A(\Delta, X)$. Let $F \subset \partial \Delta$ be a closed set of measure 0 and let $f: F \rightarrow X$ be a continuous function such that $f(s) \in \overline{\Phi(s)}(s \in G \cap F)$. Assume that $\Phi(s)$ is connected for each $s \in G \cap F$ and that the sets $\Phi(s)(s \in G \cap F)$ are equi-locally connected at the points $f(s)$. Then there exists an extension $\tilde{f} \in$ $A(\Delta, X)$ of $f$ which satisfies $\tilde{f}(z) \in \Phi(z)(z \in G-F)$.

LEMMA. Under the assumptions of the theorem with $G=\bar{\Delta}$, let $U \subset \bar{\Delta}$ be a neighbourhood of $F$ and let $\varepsilon>0$. Suppose that $F=\cup_{i=1}^{m} F_{i}$ where the $F_{i}$ $(1 \leqslant i \leqslant m)$ are pairwise disjoint nonempty closed sets. Assume that u,v: $F \rightarrow X$ are two functions such that $v(s) \in \Phi(s)(s \in F)$ and such that $u \mid F_{i}$ and $v \mid F_{i}$ are constant for each $i(1 \leqslant i \leqslant m)$. Suppose that there is some $\tilde{u} \in$ $A(\Delta, X)$ which extends $u$ and satisfies $\tilde{u}(z) \in \Phi(z)(z \in \bar{\Delta})$. Then there is an extension $\tilde{v} \in A(\Delta, X)$ of $v$ which satisfies $\tilde{v}(z) \in \Phi(z)(z \in \bar{\Delta})$ and $\| \tilde{u}(z)-$ $\tilde{v}(z) \|<\varepsilon(z \in \bar{\Delta}-U)$. If, in addition, $R>0$ and

$$
\|u(s)-f(s)\|<\delta(R), \quad\|v(s)-f(s)\|<\delta(R) \quad(s \in F)
$$

where $\delta(\cdot)$ is a modulus of equi-local connectedness of the sets $\Phi(s)(s \in F)$ at the points $f(s)$ then one may choose $\tilde{v}$ so that $\|\tilde{u}-\tilde{v}\|<4 R$.

Proof. We prove both assertions; the proof can be easily adapted to prove only the first assertion.

Observe first that by compactness of $\bar{\Delta}$, by the continuity of $\tilde{u}$ and by the fact that $\Phi$ is open there is some $\eta>0$ such that $\tilde{u}(z)+B_{\eta}(X) \subset \Phi(z)$ $(z \in \bar{\Delta})$. For the moment, fix $s \in F$. The assumptions imply that there is a path $p: I \rightarrow \Phi(s)$ such that $p(0)=u(s), p(1)=v(s)$ and $\|p(t)-f(s)\|<R$ $(t \in I)$. Since $\Phi$ is open and since $p(I)$ is compact there is some $r^{\prime}>0$ such that $p(I)+B_{r^{\prime}}(X) \subset \Phi(z)\left(z \in \bar{\Delta},|z-s|<r^{\prime}\right)$. 
Since $u$ and $v$ are constant on $F_{i}$ for $1 \leqslant i \leqslant m$ there are paths $p_{i}: I \rightarrow X$ $(1 \leqslant i \leqslant m)$ and an $r>0$ such that

$$
\begin{gathered}
p_{i}(0)=u(s), \quad p_{i}(1)=v(s) \quad\left(s \in F_{i}, 1 \leqslant i \leqslant m\right), \\
p_{i}(I)+B_{3 r}(X) \subset \Phi(z) \quad\left(z \in \bar{\Delta}, \operatorname{dist}\left(z, F_{i}\right)<r, 1 \leqslant i \leqslant m\right), \\
\operatorname{diam} p_{i}(I)<2 R \quad(1 \leqslant i \leqslant m),
\end{gathered}
$$

and $r<\min \{\varepsilon, R, \eta\}$. By the continuity of $\tilde{u}$ we may choose pairwise disjoint neighbourhoods $U_{i} \subset U$ of $F_{i}$, respectively, such that

$$
p_{i}(I)+B_{3 r}(X) \subset \Phi(z), \quad\left\|\tilde{u}(z)-p_{i}(0)\right\|<r \quad\left(z \in U_{i}, 1 \leqslant i \leqslant m\right) .
$$

By [4, Lemma 4] there exists for each $i(1 \leqslant i \leqslant m)$ an $h_{i} \in A(\Delta, X)$ such that

$$
\begin{gathered}
h_{i}\left|F_{i}=(v-u)\right| F_{i} \quad(1 \leqslant i \leqslant m), \\
h_{i} \mid F_{j}=0 \quad(1 \leqslant j \leqslant m, j \neq i), \\
h_{i}(\bar{\Delta}) \subset-p_{i}(0)+p_{i}(I)+B_{r}(X) \quad(1 \leqslant i \leqslant m) \\
\left\|h_{i}(z)\right\|<r / m \quad\left(z \in \bar{\Delta}-U_{i}, 1 \leqslant i \leqslant m\right) .
\end{gathered}
$$

Put $\tilde{v}=\tilde{u}+\sum_{i=1}^{m} h_{i}$. As in $[5$, p. 375] it is easy to see that $\tilde{v}$ has all the required properties. Q.E.D.

Proof of The TheOREM. It suffices to prove the Theorem in the case when $G=\bar{\Delta}$ (otherwise define $\Psi(z)=\Phi(z)(z \in G), \Psi(z)=X(z \in \bar{\Delta}-G)$, observe that $\Psi$ is an open map and apply the theorem to $\Psi$ ). Further, it suffices to prove the Theorem in the special case when $g=0$ (otherwise define $\Psi(z)=-g(z)+\Phi(z)(z \in G)$, observe that, by the continuity of $g, \Psi$ is open, apply the theorem to $\Psi$ and to the function $s \mapsto h(s)=-g(s)+f(s)$ and finally put $\tilde{f}=g+\tilde{h}$ ). So assume that $G=\bar{\Delta}$ and $g=0$.

Let $\delta(\cdot)$ be a modulus of equi-local connectedness of $\Phi(s)(s \in F)$ at the points $f(s)$ and let $\delta_{n}$ be a decreasing sequence of positive numbers converging to 0 and satisfying $\delta_{n}<\delta\left(\frac{1}{4} \cdot 2^{-n}\right)(n \in N)$. As in the proof of Lemma 5 , [5, pp. 371-372] it follows from our assumptions that for each $n$ there is a decomposition $F=\cup_{i=1}^{m_{n}} F_{i}$ where the $F_{i}$ are pairwise disjoint nonempty compact sets, and a function $f_{n}: F \rightarrow X$ such that $f_{n} \mid F_{i}$ is constant for each $i$ $\left(1 \leqslant i \leqslant m_{n}\right)$ and such that

(a) $f_{n}(s) \in \Phi(s)(s \in F, n \in N)$,

(b) $\left\|f_{n}(s)-f(s)\right\|<\delta_{n}(s \in F, n \in N)$.

We may assume that for all $n$, each element of $(n+1)$ st decomposition is contained in an element of $n$th decomposition. Since $\Phi$ is open, $0 \in \Phi(z)$ for all $z \in \bar{\Delta}$, and since $\bar{\Delta}$ is compact there is some $\varepsilon_{0}>0$ such that $B_{\varepsilon_{0}}(X) \subset$ $\Phi(z)(z \in \bar{\Delta})$. Let $U_{n} \subset \bar{\Delta}$ be a decreasing sequence of neighbourhoods of $F$ 
such that $F=\cap{ }_{n=1}^{\infty} U_{n}$. Assume that there exist a decreasing sequence $\varepsilon_{n}$ of positive numbers satisfying $\varepsilon_{n}<\varepsilon_{0}(n \in N)$ and a sequence $g_{n} \in A(\Delta, X)$, $g_{0}=0$, with the following properties:

(i) $g_{n}\left|F=f_{n}\right| F(n \in N)$,

(ii) $\left\|g_{n}-g_{n-1}\right\|<1 / 2^{n-1}(n \in N, n \geqslant 2)$,

(iii) $\left\|g_{n}(z)-g_{n-1}(z)\right\|<\varepsilon_{n-1} / 2^{n}\left(z \in \bar{\Delta}-U_{n}, n \in N\right)$,

(iv) $g_{n}(z)+B_{\varepsilon_{n}}(X) \subset \Phi(z)(z \in \bar{\Delta}, n \in N)$.

By (ii), $g_{n}$ converge uniformly on $\bar{\Delta}$ so putting $\tilde{f}(z)=\lim _{n \rightarrow \infty} g_{n}(z)(z \in \bar{\Delta})$ we have $\tilde{f} \in A(\Delta, X)$. By (i) and (b) $\tilde{f} \mid F=f$. Further, let $z \in \bar{\Delta}-U_{1}$. Writing $\tilde{f}(z)=\sum_{n=0}^{\infty}\left(g_{n+1}(z)-g_{n}(z)\right)$ it follows by (iii) that $\tilde{f}(z) \in B_{\varepsilon_{0}}(X) \subset \Phi(z)$. Finally, if $z \in U_{1}-F$ then for some $n \in N$ we have $z \notin U_{j}(j>n)$ so by (iii) and (iv) it follows that

$$
\tilde{f}(z)=g_{n}(z)+\sum_{j=n}^{\infty}\left[g_{j+1}(z)-g_{j}(z)\right] \in g_{n}(z)+B_{e_{n}}(X) \subset \Phi(z)
$$

and consequently $\tilde{f}$ has all the required properties.

It remains to prove the existence of $\varepsilon_{n}$ and $g_{n}$ with the above properties. Put $g_{0}=0$. By the first part of the Lemma there is some $g_{1} \in A(\Delta, X)$ satisfying $g_{1}(z) \in \Phi(z)(z \in \bar{\Delta})$ and such that (i) and (iii) hold for $n=1$. Since $\Phi$ is open, $g_{1}(z) \in \Phi(z)$ for all $z \in \bar{\Delta}$ and since $\bar{\Delta}$ is compact there is some $\varepsilon_{1}$ with $0<\varepsilon_{1}<\varepsilon_{0}$ such that $g_{1}(z)+B_{\varepsilon_{1}}(X) \subset \Phi(z)(z \in \bar{\Delta})$. Let $m \in N$ and assume that $g_{m} \in A(\Delta, X)$ satisfies (i) and (iv) for some $\varepsilon_{m}>0$. By the Lemma (a) and (b) imply that there is some $g_{m+1} \in A(\Delta, X)$ satisfying (i)-(iii) for $n=m+1$ and such that $g_{m+1}(z) \in \Phi(z)(z \in \bar{\Delta})$. Again, since $\Phi$ is open, $g_{m+1}(z) \in \Phi(z)$ $(z \in \bar{\Delta})$ and since $\bar{\Delta}$ is compact there is some $\varepsilon_{m+1}$ with $0<\varepsilon_{m+1}<\varepsilon_{m}$ such that $g_{m+1}(z)+B_{\varepsilon_{m+1}}(X) \subset \Phi(z)(z \in \bar{\Delta})$. Q.E.D.

Next we present some simple applications. In Corollaries 1-6 below $G$ can be either $\bar{\Delta}$ or $\partial \Delta$.

Corollary 1. Let $X$ be a complex Banach space and let $p: G \rightarrow(0, \infty)$ be a lower semicontinuous function. Given any closed set $F \subset \partial \Delta$ of measure 0 and any continuous function $f: F \rightarrow X$ satisfying $\|f(s)\| \leqslant p(s)(s \in F)$ there exists $\tilde{f} \in A(\Delta, X)$ which extends $f$ and satisfies $\|\tilde{f}(z)\|<p(z) \quad(z \in G-F)$. Moreover, if $z_{j} \in \Delta(1 \leqslant j \leqslant k)$ and if $n_{j}(1 \leqslant j \leqslant k)$ are positive integers, $\tilde{f}$ can be chosen to have a zero at $z_{j}$ of order at least $n_{j}$.

Proof. Since $p$ is lower semicontinuous the map $z \mapsto \Phi(z)=\{x \in X$ : $\|x\|<p(z)\}$ is open on $G$. Further, since $p$ is lower semicontinuous there is some $\delta>0$ such that $p(z) \geqslant \delta(z \in G)$. Consequently $g \in A(\Delta, X)$ defined by $g(z)=0(z \in \bar{\Delta})$ satisfies $g(z) \in \Phi(z)(z \in G)$. Further, it is easy to see that any family $\left\{P_{\alpha} ; \alpha \in \mathbb{Q}\right\}$ of nonempty open convex subsets of $X$ is equi-locally connected at the points $x_{\alpha}$ for any $x_{\alpha} \in \bar{P}_{\alpha}(\alpha \in \mathbb{Q})$ so the sets 
$\Phi(s)(s \in F)$ are equi-locally connected at $f(s)$. Now the first assertion follows by the Theorem. To prove the second assertion, multiply $\tilde{f}$ by $\varphi \in A$ which satisfies $\varphi|F=1,| \varphi(z) \mid \leqslant 1(z \in \bar{\Delta})$ and has a zero at $z_{j}$ of order at least $n_{j}$ [2, Theorem, pp. 284-285]. Q.E.D.

Corollary 1 sharpens and generalizes [2, Theorem, pp. 284-285]. Note that in the case when $X=C$ Corollary 1 is an easy consequence of [6, Theorem $3]$.

Corollary 2. Let $X$ be a complex Banach space and let $P \subset X$ be a nonempty open connected set which is locally connected at every point of $\bar{P}$. Let $\varphi: G \rightarrow C$ be a continuous function such that $\varphi(z) g(z) \in P(z \in G)$ for some $g \in A(\Delta, X)$. Given any closed set $F \subset \partial \Delta$ of measure 0 and any continuous function $f: F \rightarrow X$ satisfying $\varphi(s) f(s) \in \bar{P}(s \in F)$ there exists $\tilde{f} \in A(\Delta, X)$ which extends $f$ and satisfies $\varphi(z) \tilde{f}(z) \in P(z \in G-F)$.

Proof. Define $\Phi(z)=\{x \in X: \varphi(z) x \in P\}(z \in G)$. Since $P$ is open and since $\varphi$ is continuous $\Phi$ is an open map; since $P$ is connected $\Phi(z)$ is connected for each $z \in G$. By the continuity of $\varphi$ and $f$ the set $S=$ $\{\varphi(s) f(s), s \in F\} \subset \bar{P}$ is compact and consequently $P$ is uniformly locally connected on $S$ [5]. Since $\varphi$ is bounded on $F$ it follows easily that the sets $\Phi(s)(s \in F)$ are equi-locally connected at the points $f(s)$. Now the assertion follows by the Theorem. Q.E.D.

Similarly we prove

Corollary 3. Let $X$ be a complex Banach space and let $P \subset X$ be a nonempty open connected set which is locally connected at every point of $\bar{P}$. Let $h: G \rightarrow X$ be a continuous function such that $h(z)+g(z) \subset P(z \in G)$ for some $g \in A(\Delta, X)$. Given any closed set $F \subset \partial \Delta$ of measure 0 and any continuous function $f: F \rightarrow X$ satisfying $h(s)+f(s) \in \bar{P}(s \in F)$ there exists $\tilde{f} \in A(\Delta, X)$ which extends $f$ and satisfies $h(z)+\tilde{f}(z) \in P(z \in G-F)$.

Next we present some dominated extension theorems for the disc algebra.

Corollary 4. Let $p: G \rightarrow[0, \infty)$ be an upper semicontinuous (USC) function and let $q: G \rightarrow(0, \infty)$ be a lower semicontinuous (LSC) function such that $p(z)<|g(z)|<q(z)(z \in G)$ for some $g \in A$. Given any closed set $F \subset \partial \Delta$ and any continuous function $f: F \rightarrow C$ satisfying $p(s)<|f(s)|<q(s)$ $(s \in F)$ there is an $\tilde{f} \in A$ which extends $f$ and satisfies $p(z)<|f(z)|<q(z)$ $(z \in G-F)$.

Proof. Define $\Phi(z)=\{\zeta \in C: p(z)<|\zeta|<q(z)\} \quad(z \in G)$. Let $z_{0} \in G$ and let $\zeta_{0} \in \Phi\left(z_{0}\right)$. For some $\varepsilon>0$ we have $p\left(z_{0}\right)+\varepsilon<|\eta|<q\left(z_{0}\right)-\varepsilon$ for all $\eta \in \zeta_{0}+B_{\varepsilon}(C)$. Since $p$ is USC and since $q$ is LSC there is a 
neighbourhood $U \subset G$ of $z_{0}$ such that $p(z)<p\left(z_{0}\right)+\varepsilon, q(z)>q\left(z_{0}\right)-\varepsilon$ $(z \in U)$ and consequently $\Phi$ is open on $G$. Clearly $\Phi(z)$ is connected for every $z \in G$. Let $0 \leqslant r<R$ and let $S=\{\zeta \in C: r<|\zeta|<R\}$. It is easy to see that $\left(z+B_{\varepsilon}(C)\right) \cap S$ is connected for every $z \in S$ and for every $\varepsilon>0$. Consequently the sets $\Phi(s)(s \in F)$ are equi-locally connected at the points $f(s)$. Now the assertion follows by the theorem. Q.E.D.

Remark. To prove Corollary 4 in the case when $G=\partial \Delta$ one needs to assume only that $p(z)<q(z)(z \in G)$ and one does not need the existence of $g \in A$. Namely, by [10, Theorem 5.3, p. 15] there exists a continuous function $\varphi: G \rightarrow R$ satisfying $p(z)<\varphi(z)<q(z)(z \in G)$ and since $G$ is compact there is some $\varepsilon>0$ such that $p(z)+\varepsilon<\varphi(z)<q(z)-\varepsilon(z \in G)$. By $[11$, p. 216] $A$ approximates in modulus on $\partial \Delta$ so there is some $g \in A$ such that $\varphi(z)-\varepsilon<|g(z)|<\varphi(z)+\varepsilon(z \in G)$ and consequently $p(z)<|g(z)|<$ $q(z)(z \in G)$.

Corollary 5. Let $p_{1}, q_{1}: G \rightarrow R$ be two upper semicontinuous functions and let $p_{2}, q_{2}: G \rightarrow R$ be two lower semicontinuous functions such that $p_{1}(z)<$ $\operatorname{Re} g(z)<p_{2}(z), q_{1}(z)<\operatorname{Im} g(z)<q_{2}(z)(z \in G)$ for some $g \in A$. Given any closed set $F \subset \partial \Delta$ of measure 0 and any continuous function $f: F \rightarrow C$ satisfying $p_{1}(s) \leqslant \operatorname{Re} f(s) \leqslant p_{2}(s), q_{1}(s) \leqslant \operatorname{Im} f(s) \leqslant q_{2}(s)(s \in F)$ there exists an $\tilde{f} \in$ $A$ which extends $f$ and satisfies $p_{1}(z)<\operatorname{Re} f(z)<p_{2}(z), q_{1}(z)<\operatorname{Im} f(z)<$ $q_{2}(z)(z \in G-F)$.

Proof. Define $\Phi(z)=\left\{\zeta \in C: p_{1}(z)<\operatorname{Re} \zeta<p_{2}(z), q_{1}(z)<\operatorname{Im} \zeta<\right.$ $q_{2}(z)(z \in G)$ and observe that by the semicontinuity of $p_{1}, p_{2}, q_{1}, q_{2} \Phi$ is open on $G$. Further, since $\Phi(z)$ is convex for every $z \in G$ it follows that the sets $\Phi(s)(s \in F)$ are equi-locally connected at the points $f(s)$. Now the assertion follows by the Theorem. Q.E.D.

Corollary 6. Let $p: G \rightarrow(0, \infty)$ be a lower semicontinuous function. Given any closed set $F \subset \partial \Delta$ of measure 0 and any continuous function $f: F \rightarrow C$ satisfying $|f(s)| \leqslant p(s), \operatorname{Re} f(s) \geqslant 0(s \in F)$ there exists an $\tilde{f} \in A$ which extends $f$ and satisfies $|f(z)|<p(z), \operatorname{Re} f(z)>0(z \in G-F)$.

Proof. Define $\Phi(z)=\{\zeta \in C:|\zeta|<p(z), \operatorname{Re} \zeta>0\}(z \in G)$. Since $p$ is LSC, $\Phi$ is open on $G$. Since $p$ is LSC and positive and since $G$ is compact there is some $\delta>0$ such that $p(z) \geqslant \delta(z \in G)$. Define $g \in A$ by $g(z)=$ $\delta / 2(z \in \bar{\Delta})$. Clearly $g(z) \in \Phi(z)(z \in G)$. Since $\Phi(z)$ is convex for every $z \in G$ it follows that the sets $\Phi(s)(s \in F)$ are connected and equi-locally connected at the points $f(s)$. Now the assertion follows by the Theorem. Q.E.D.

Corollary 6 with $G=\partial \Delta$ sharpens [7, Corollary 4.5]. Corollary 6 with $G=\bar{\Delta}$ answers a question in [7, p. 294]. 


\section{REFERENCES}

1. E. Bishop, A general Rudin-Carleson theorem, Proc. Amer. Math. Soc. 13 (1962), 140-143.

2. T. W. Gamelin, Restrictions of subspaces of $C(X)$, Trans. Amer. Math. Soc. 112 (1964), 278-286.

3. _ Uniform algebras, Prentice-Hall, Englewood Cliffs, N. J., 1969.

4. J. Globevnik, Analytic extensions of vector-valued functions, Pacific J. Math. 63 (1976), 389-395.

5.

6. P. Hag, A generalization of the Rudin-Carleson theorem, Proc. Amer. Math. Soc. 43 (1974), 341-344.

7. 283-294.

8. E. Michael, Selected selection theorems, Amer. Math. Monthly 63 (1956), 233-238.

9. Continuous selections. II, Ann. of Math. (2) 64 (1956), 562-580.

10. Z. Semadeni, Simultaneous extensions and projections in spaces of continuous functions, Lecture Notes, Univ. of Aarhus, 1965.

11. E. L. Stout, The theory of uniform algebras, Bogden and Quigley, Tarrytown-on-Hudson, N. Y., 1971.

Institute of Mathematics, Physics and Mechanics, University of Lubluana, LJubluna, YugosLavia

Current address: Department of Mathematics, University of Washington, Seattle, Washington 98195 